\title{
The influence of fibre length, diameter and concentration on the strength and strain to failure of glass-fibre reinforced Polyamide 6,6.
}

\section{J. L. Thomason}

University of Strathclyde, Department of Mechanical Engineering, 75 Montrose Street, Glasgow G1 1XJ, United Kingdom.

Keywords: A Polymer-matrix composites (PMCs), B Mechanical properties, E Injection moulding

\footnotetext{
Abstract

Results of an investigation of the mechanical performance of injection moulded long glass fibre reinforced polyamide 6,6 composites are presented. The glass fibre content in these composites was varied over the range $10-50 \%$ by weight using fibres with average diameters of 10,14, and 17 micrometres. Mechanical testing and analysis of the apparent interfacial shear strength was carried out at $23^{\circ} \mathrm{C}$ and $150^{\circ} \mathrm{C}$ on dry-asmoulded and boiling water conditioned samples. The results from these composites are compared with standard extrusion compounded short glass fibre materials. The influence of fibre diameter and concentration on the residual fibre length, fibre orientation distribution and composite strength and elongation to failure is presented and discussed in comparison to the predictions of some of the available micromechanical models.
} 


\section{Introduction}

In recent years there has been strong growth in the use of long glass fibre thermoplastic composite systems in semi-structural and engineering applications. These thermoplastic matrix composite systems combine ease of processing with property advantages such as enhanced toughness and an unlimited shelf life. Furthermore, their intrinsic recyclability is rapidly being recognised as a strong driving force for their further application. Their potential for high-volume processing combined with high levels of end use performance levels and associated lower manufacturing costs has spurred the current expansion of research and development activities on thermoplastic matrix composites. Glass fibre reinforced polyamides are excellent composite materials in terms of their high levels of mechanical performance and temperature resistance. The mechanical performance of these composites results from a combination of the fibre and matrix properties and the ability to transfer stresses across the fibre-matrix interface. Variables such as the fibre content, diameter, orientation and the interfacial strength are of prime importance to the final balance of properties exhibited by injection moulded thermoplastic composites [17].

Short fibre reinforced thermoplastics have been used in the automotive industry for many years and there has recently been a strong growth in the use of polyamide based materials in under-the-hood applications [8]. More recently there has been an increasing growth in the use of long fibre thermoplastic composite systems in semistructural and engineering applications. It is interesting to note that the growth rates for polypropylene based long fibre compounds has far exceeded that of other long fibre thermoplastic systems over the last decade. This has occurred despite the fact that many of the early developments and long fibre thermoplastic products were based on polyamide resins [9-12]. It may well be that part of the background to this phenomenon 
lies in the excellent levels of profitability, processibility, and performance of these materials. Achieving the correct balance of these "3P's" is critical to the success of any product in its appropriate market. Notwithstanding these facts there has been considerable discussion recently that the next major long fibre development may be in thermoplastic systems based on higher performance resins than polypropylene. Glass fibre reinforced polyamides are excellent composite materials however the mechanical properties of polyamide based composites decrease markedly upon the absorption of water and other polar fluids [13-15]. There also exist a number of well documented differences in the structure performance relationships of short fibre reinforced polyamide and polypropylene composites and it can be expected that there will also be differences when comparing these resins reinforced with long fibres.

In this report data are presented on the mechanical performance of long fibre reinforced polyamide 6,6 which may be relevant to the above discussion. Injection moulded long fibre reinforced polyamide 6,6 samples have been prepared with a range of glass contents $(0-50 \% \mathrm{wt})$ and two sizing chemistries for polyamide reinforcement. These long fibre compounds have been produced with glass fibres having average fibre diameters of 10,14, and $17 \mu \mathrm{m}$. Mechanical performance has been determined for both the "dry as moulded" state $(\mathrm{DaM})$ and after hydrolytic and temperature conditioning and compared with reference short fibre composites based on $10 \mu \mathrm{m}$ diameter fibre in the same resin system. Data on the influence of the above variables on the residual fibre length and fibre orientation distribution in the moulded composites and the composite modulus have been presented previously [16]. In this paper data on the influence of the above conditioning environments and micromechanical parameters on the composite strength and tensile elongation to failure are presented and discussed. The results on composite impact performance will be published in a subsequent paper. 


\section{Experimental}

The glass samples used for the production of the long glass fibre pellets were continuous Advantex ${ }^{\circledR}$ glass (boron free E-glass) Type $30^{\circledR}$ packages produced on a single production bushing. The glass was coated with sizing formulation R43S, which is a polyamide compatible sizing optimized for continuous glass products. Samples (LF10, LF14, LF17) were produced with nominal fibre diameters of $10,14,17 \mu \mathrm{m}$ and linear density (tex) of 1200, 2400, $3500 \mathrm{~g} / \mathrm{km}$ as shown in Table 1 . A reference short fibre compound (SF10) was produced using DS1123 a nominal $10 \mu \mathrm{m}$ fibre diameter chopped glass product coated with polyamide sizing optimized for chopped glass production. The polyamide 6,6 (PA6,6) used for composite production was DuPont Zytel 101. Reference samples of the unreinforced resin were also included. Given that the matrix in the fibre reinforced materials experiences an extra heat cycle, reference resin samples were moulded from resin which had been run one time through the extruder with the same temperature profile as applied during the composite pellet production step.

Long fibre reinforced pellets were produced using a standard pultrusion type process [16] where the continuous glass was fed into an impregnation unit consisting of a heated oblong box containing a number of spreader bars and a circular exit die of fixed diameter. The impregnation unit was attached to, and fed by, a single screw extruder which delivered polymer melt to the unit at a rate appropriate to the pulling speed of the glass $(30 \mathrm{~m} / \mathrm{min})$ and the desired final glass:resin ratio of the pellets. The temperature of the molten resin was maintained between $300-310^{\circ} \mathrm{C}$ in the impregnation unit. After exiting the die the resin impregnated glass was cooled in a water bath before passing through a pulling and chopping operation. Nominal pellet chop length was $12.5 \mathrm{~mm}$. For the short

fibre compound, the chopped glass bundles and pre-dried PA6,6 pellets were dry blended by weight to the appropriate glass content and compounded on a single screw extruder 
(2.5 inch, 3.75:1, 24:1 L/D screw). Set point temperatures were $288-293^{\circ} \mathrm{C}$ for compounding. The compounds were moulded into test bars on a 200-ton Cincinnati Milacron moulding machine. Set point temperatures were $293-299^{\circ} \mathrm{C}$ for moulding, at a mould temperature of $93^{\circ} \mathrm{C}$.

Tensile properties were measured in accordance with the procedures in ASTM D-638, using ASTM Type I specimens at a crosshead rate of $5 \mathrm{~mm} / \mathrm{min}(0.2$ inches $/ \mathrm{min})$ and an extensometer gauge length of $50 \mathrm{~mm}$ ( 2 inches). Tensile properties were measured "dry as moulded" (DaM) at $23^{\circ} \mathrm{C}$ and $150^{\circ} \mathrm{C}$ and at $23^{\circ} \mathrm{C}$ after 24 hour boiling water conditioning. DaM flexural properties were measured only at $23^{\circ} \mathrm{C}$ in accordance with the procedures in ASTM D-790, at a crosshead rate of $2.5 \mathrm{~mm} / \mathrm{min}(0.1 \mathrm{inches} / \mathrm{min})$ and a span width of $50 \mathrm{~mm}$ ( 2 inches). Unless otherwise stated, all mechanical property testing was performed at $23^{\circ} \mathrm{C}$ and at a relative humidity of $50 \%$. Fibre length, diameter, and orientation distributions were determined by image analysis and optical microscopy as previously described [16].

\section{Results}

A summary of a previously published [16] characterization of the samples in this study is presented in Table 1. The data for composite strength presented in Figures 1 and 2 show a clear dependence on both fibre content and diameter. In both Figures it can be seen that increasing the fibre content results in an increase in composite strength over the range of the study. For tensile strength the greatest increase comes in the range 20$40 \% \mathrm{wt}$, and above $40 \% \mathrm{wt}$ reinforcement there appears to be a levelling off in the strength improvement. With regard to fibre diameter, it is clear that finer fibres bring a higher reinforcement level in the $20-40 \% \mathrm{wt}$ range. There is some evidence of a 
crossover at lower glass contents in tensile strength and that the finer fibres may not have as high a reinforcement efficiency as the thicker fibres. Although this conclusion depends strongly on the single data point at $12 \% \mathrm{wt}$, it can be seen that the trend line for the LF10 samples would seem to indicate that this data point is acceptable. Clearly there is scope for further investigation at lower fibre contents. It is also interesting to note that the advantage of the finer fibres appears to disappear at glass contents above $40 \%$ wt. In particular the flexural strength data for LF10, LF14, and L17 appear to be converging to a single line above $40 \%$ wt. Although the tensile and flexural strength data appear to follow similar trends there is a significant difference in their magnitude. This is illustrated in Figure 3 which shows the flexural strength plotted directly against the tensile strength for all samples. It can be seen that virtually all samples in this study, including the unreinforced resin samples, fall on a single lie. The slope of this line indicates a direct relationship of flexural strength approximately equal to 1.6 times the tensile strength.

Figure 4 summarises the results for the tensile strength of the boiling water conditioned samples. In Figure 4 the plasticizing effect of water on PA6,6 and its composites is clearly revealed, the results for tensile strength after boiling water conditioning show a large reduction in comparison to the DaM results. In this case the reduction in performance is in the range of $30-50 \%$ for the composite samples and $50 \%$ loss in the tensile strength of the resin. The SF10 reference sample exhibited a slightly greater loss in tensile strength that the equivalent LF10 sample and consequently there is a greater advantage of LF over SF apparent in these results. With regard to the effect of fibre diameter it can seen from Figure 4 that the ranking of finer fibre giving higher tensile strength is clearly maintained through the boiling water conditioning. The results for the tensile strength tested on dry samples at $150^{\circ} \mathrm{C}$ in Figure 5 reveal similar trends to the 
data obtained after boiling water conditioning. Tensile strength measured at $150^{\circ} \mathrm{C}$ exhibited a large reduction in comparison with DaM test results at $23^{\circ} \mathrm{C}$.

The results for DaM tensile elongation at $23^{\circ} \mathrm{C}$ shown in Figure 6 are quite complex. The addition of a small fraction of reinforcement substantially lowers the tensile elongation of the system from that of the PA6,6 resin. It appears that the depth of this drop is dependent on fibre diameter with finer fibre causing a greater loss in elongation than thicker fibres. As the fibre content is increased $(>20 \% \mathrm{wt})$ a recovery in the composite elongation is observed with the finer fibre apparently showing the greatest increase. The tensile elongation then appears to reach a maximum in the $30-40 \% \mathrm{wt}$ fibre level and above $40 \% \mathrm{wt}$ a gradual decline in elongation with increasing glass content is observed. There is some evidence that the elongation values for different fibre diameters also appear to be converging above $40 \%$ wt fibre. The results for tensile elongation after boiling water conditioning are shown in Figure 7. These data also show the plasticising effect of this conditioning on the polyamide matrix and the potential loss of interfacial bonding in these composites when compared with the DaM results in Figure 6. The composite elongation shows a large increase after conditioning, particularly in the LF samples with higher resin content. Furthermore, a dependence on fibre length can be noted since the SF10 reference sample shows a particularly large increase in comparison with the LF10 equivalent glass content sample. It can also be noted that the shape of the curve of elongation vs fibre content is very different for the conditioned samples compared with the DaM samples in Figure 6. The results for the tensile elongation of samples tested dry at $150^{\circ} \mathrm{C}$ in Figure 8 reveal similar trends to the data obtained after boiling water conditioning. 


\section{Discussion}

It has been well documented recently that for improved mechanical performance of discontinuous fibre reinforced thermoplastics, in general, longer residual fibre length is better $[7,9,12,16,17]$. However, it is clear from the results in Table 1 that, when dealing with the injection moulding process, the residual fibre length is not a property that can be directly controlled in the final composite part. The fibre length in the moulded composite is a property which is influenced by the input fibre length, the composite fibre content and the diameter of fibres employed. This may well be due to the fact that decreased average fibre diameter at equal fibre loading, or increased fibre content at equal fibre diameter, leads to a decreased average fibre-fibre spacing and consequently an increased probability of fibre-fibre and fibre-machine interaction with resultant fibre damage and breakage. This decreased fibre-fibre spacing also leads to an increased apparent melt viscosity resulting in higher bending forces on the fibres during moulding. Furthermore dispersion of the reinforcement to individual fibres becomes more of a challenge at increased fibre content and the possibility of fibre agglomeration becomes greater. It is interesting to note that when the data are examined in terms of residual fibre aspect ratio (length/diameter), the data for the various LF compounds collapse onto a single line [16]. In the commercially important fibre content range of $30-40 \%$ the LF compounds deliver a residual fibre aspect ratio after moulding approximately $50 \%$ higher compared to the SF compounds.

Reliable analysis of the relationship between tensile strength and the microstructural parameters of injection moulded fibre reinforced thermoplastic composites requires the experimental characterization of a large number of parameters. These include the fibre volume fraction, the fibre length distribution, the fibre diameter distribution, the fibre orientation distribution, the fibre strength-length relationship, the stress transfer 
capability of the fibre-matrix interface and the contribution of the polymer matrix [119]. This situation is further complicated by the fact that many of these parameters (fibre length, diameter and orientation) vary over a wide range within an injection moulded part. Considerable experimental effort has been expended in this study to characterize these distributions for all samples involved [16]. However a value for the interfacial shear strength characterizing the stress transfer capability of the fibre-matrix interface is still required.

The macro-method analysis used here to obtain values of the apparent interfacial shear strength (IFSS) was originally proposed by Bowyer and Bader [20,21] and an improved version has been extensively reviewed by Thomason [13,22-24]. The macro-method has a significant attraction over some other methods in that it utilizes data which are readily available from standard composite mechanical testing and requires only an extra determination of fibre length distribution, which is a common characterisation tool of those working with discontinuous fibre composites. The method is based on the Kelly-Tyson model for the prediction of the strength $\left(\sigma_{\mathrm{uc}}\right)$ of a polymer composite reinforced with discrete aligned fibres [25]. This model can be simplified to $\sigma_{\mathrm{uc}}=\eta_{\mathrm{o}}(\mathrm{X}$ $+\mathrm{Y})+\mathrm{Z}$, where $\mathrm{Z}$ is the matrix contribution, $\mathrm{X}$ is the sub-critical fibre contribution, and $\mathrm{Y}$ is the super critical contribution, in reference to a critical fibre length defined by $\mathrm{L}_{c}=$ $\sigma_{\mathrm{uf}} \mathrm{D} / 2 \tau$ where $\sigma_{\mathrm{uf}}$ is the fibre strength, $\mathrm{D}$ is the average fibre diameter and $\tau$ is the IFSS. The Kelly-Tyson model assumes that all the fibres are aligned in the loading direction and the equation cannot be integrated to give a simple numerical orientation factor to account for the average fibre orientation. When the stress at the $0.5 \%$ and $1 \%$ strain levels obtained from tensile testing are combined with the full fibre length distributions used to obtain the averages in Table 1 and applied in the procedure described above values for the parameters $\eta_{\mathrm{o}}$ and $\tau$ may be obtained. In common with 
the previous discussion on orientation factors [16], the values obtained for $\eta_{\mathrm{o}}$ by this method exhibited no obvious correlations with fibre diameter or fibre concentration and could be averaged to the value $0.72 \pm 0.02$ which is comparable with the other values obtained by optical microscopy [16].

Results obtained for the apparent IFSS in injection moulded short glass fibre reinforced PA6,6 have previously shown a strong inverse relationship between IFSS and average fibre diameter at 33\% glass content [14] and a moderate inverse dependence of IFSS on fibre content for $10 \mu \mathrm{m}$ average fibre diameter [13]. The results for the IFSS obtained using the macro-method on these LF samples are shown in Figure 9. The data for the LF samples appear to confirm the previously observed SF trend for a moderate decrease in IFSS with increasing fibre content. It has been shown previously [13,22-24] that this relationship can be explained by the fact that a fraction of the apparent IFSS is due to the residual radial compressive stresses at the fibre matrix interface in these thermoplastic composites. These stresses decrease with increasing fibre content. The previously obtained range of IFSS obtained for 33\% SF-PA6,6 composites with a similar range of fibre diameter is also indicated in Figure 9 and also appears to agree well with the current LF trends. It can be postulated that the apparent IFSS in these composites should not exceed the shear stress of the PA6,6 matrix which can be estimated from the tensile strength to be approximately $45 \mathrm{MPa}$. It should be noted that this discussion ignores the many possible reasons why the properties of the resin close to the fibre could well be different from those of a moulded bulk resin sample. It appears that, in general, the data shown in Figure 9 are levelling off at low fibre content at or below this $45 \mathrm{MPa}$ value which confirms the commonly accepted idea that fibre to polyamide adhesion levels are close to the maximum possible in the DaM state with most of the current glass fibre sizing systems available for polyamide reinforcement. 
Consequently it can be suggested that improvements to DaM performance of glass reinforced polyamide can more profitably be sought in structure-performance parameters other than adhesion.

Figure 10 shows the results for a similar analysis on the IFSS in the boiling water conditioned samples. The Figure reveals similar trends as those observed in Figure 9 for the DaM samples, albeit with a somewhat greater level of scatter which might be expected from the potential extra variability from small differences in the level of water absorption and matrix plasticization of individual test samples. Figure10 still shows a significant trend for reduction in apparent IFSS with increasing fibre content. The absolute level of IFSS in the boiled samples is significantly lower than that of the DaM samples and also appears to be approaching an upper limit close to the shear strength of the matrix calculated from the tensile strength as approximately $23 \mathrm{MPa}$.

The above analysis relies on the use of the Kelly-Tyson model to analyse the stressstrain relationship in the composite prior to failure. Of course the same model is often used to analyse experimental data on the tensile strength of such composites. Although the model was originally developed for aligned discontinuous fibre composites it is often presented with an additional, empirically obtained, orientation factor $\left(\eta_{\mathrm{o}}\right)$ as shown below

$$
\sigma_{c}=\eta_{\mathrm{o}}\left(\sum_{\mathrm{i}}\left[\frac{\tau \mathrm{L}_{\mathrm{i}} \mathrm{V}_{\mathrm{i}}}{\mathrm{D}}\right]+\sum_{\mathrm{j}}\left[\mathrm{E}_{\mathrm{f}} \varepsilon_{\mathrm{c}} \mathrm{V}_{\mathrm{j}}\left(1-\frac{\mathrm{E}_{\mathrm{f}} \varepsilon_{\mathrm{c}} \mathrm{D}}{4 \tau \mathrm{L}_{\mathrm{j}}}\right)\right]\right)+\left(1-\mathrm{V}_{\mathrm{f}}\right) \mathrm{E}_{\mathrm{m}} \varepsilon_{\mathrm{c}}
$$

By rearrangement of this equation the following version is obtain

$$
\sigma_{\mathrm{c}}-\left(1-\mathrm{V}_{\mathrm{f}}\right) \mathrm{E}_{\mathrm{m}} \varepsilon_{\mathrm{c}}=\eta_{\mathrm{o}}\left(\sum_{\mathrm{i}}\left[\frac{\tau \mathrm{L}_{\mathrm{i}} \mathrm{V}_{\mathrm{i}}}{\mathrm{D}}\right]+\sum_{\mathrm{j}}\left[\mathrm{E}_{\mathrm{f}} \varepsilon_{\mathrm{c}} \mathrm{V}_{\mathrm{j}}\left(1-\frac{\mathrm{E}_{\mathrm{f}} \varepsilon_{\mathrm{c}} \mathrm{D}}{4 \tau \mathrm{L}_{\mathrm{j}}}\right)\right]\right)
$$


Using equation 2 it is possible to obtain $\eta_{\mathrm{o}}$ from the experimental data by examining the fibre contribution to the composite strength as a function of fibre volume fraction. The results of such an analysis for both DaM and boiled samples are shown in Figure 11. It can be seen that both data sets fall on or around the same line with a small amount of scatter. This should be as expected since the boiling water conditioning should not cause a great deal of change in the average fibre orientation distribution in the composites. A least squares analysis, forced through the origin, predicts a value of $\eta_{0}=0.69$. This is significantly higher than the value of 0.59 obtained [26] across a range of LFPP composites averaged across a similar glass content to this study of LFPA. However, an almost identical value of $\eta_{\mathrm{o}}=0.70$ was obtained in a recent study of SFPA properties versus glass fibre content [13]. It may be concluded that the value of the orientation parameter used in the Kelly-Tyson equation may be dependent on the properties of the composite matrix as well as the fibre orientation distribution in these injection moulded materials.

A recent study [13] of the influence of fibre diameter on the mechanical performance of injection moulded short-fibre reinforced PA6,6 reported a small, but significant, loss of tensile strength of approximately $-1.7 \%$ per one $\mu \mathrm{m}$ increase in average fibre diameter in the range of $10-17 \mu \mathrm{m}$ at a $33 \% \mathrm{wt}$ glass content. In Figure 12 the data from these LF samples are examined in a similar manner. The figure shows the diameter dependence of the tensile strength from the LF samples at three fixed fibre contents and compares these data with those previously obtained for SF samples. Although the LF samples also exhibit a significant dependence of DaM tensile strength on average fibre diameter the effect is clearly less than that observed with the SF sample study of fibre diameter effects. The slope of the least squares fitted lines in Figure 12 indicate that the LF samples dependence on fibre diameter is approximately half that of the SF samples. 
Figure 13 presents the diameter dependence of the sample tensile strength after boiling water conditioning. In this case there appears to be a greater drop off in tensile strength with increasing diameter. The slopes of the lines in Figure 13 also reveal a trend for increasing fibre diameter dependence with decreasing fibre content. Comparing the LF data at 30\%wt with the previous SF data at 33\%wt it can be seen that the lines have similar slopes. The diameter dependence of the flexural strength is shown in Figure 14. The diameter dependence of the flexural strength appears to be somewhat greater than that of the tensile strength; however a similar trend of decreasing sensitivity to fibre diameter with increasing fibre content can also be observed in Figure 14.

Similar results for a higher composite flexural strength compared to tensile strength observed in Figure 3 have been reported in unidirectional continuous fibre reinforced composites [27]. The phenomenon has been explained in terms of Weibull statistical strength theory. Strength is assumed to be controlled by critical defects which are statistically distributed. In a tensile test a much higher volume of material is subject to the maximum stress than in a flexural test. Consequently the chances of a critical flaw are higher and the tensile strength is therefore lower. It has been reported [27] that the ratio of strength between pure bending $\left(\sigma_{\mathrm{B}}\right)$ and pure tension $\left(\sigma_{\mathrm{T}}\right)$ for specimens of equal volume is

$$
\frac{\sigma_{B}}{\sigma_{T}}=[2(m+1)]^{1 / m}
$$

where $\mathrm{m}$ is the Weibull modulus related to the strength variability. A ratio of 1.6 is equivelant to $\mathrm{m}=5.5$. 
The complex behaviour of the tensile elongation to failure of the LF samples also merits further note here. It has been shown in this study that the tensile strength of LFPA appears to follow basically similar trends to SFPA with regards to fibre concentration and diameter albeit at a different absolute level of performance. The DaM elongation behaviour of LFPA exhibits very different trends to SFPA with regards to fibre concentration. The data in Figure 6 appear to show a transition in the tensile elongation behaviour of the specimens at approximately $35 \%$ of fibre. In Figure 15 we contrast the behaviour of the LF10 samples with previously published data on DaM short fibre reinforced PA6,6 samples where the fibre diameter was also $10 \mu \mathrm{m}$. The SF samples exhibited a steep drop in tensile elongation between $0-10 \%$ fibre content and then a further more gradual decrease as the glass content is further increased. At fibre contents above $35 \%$ the LF data from this study coincide well with this SF data. However, below $35 \%$ fibre content the LF results show a progressively more brittle behaviour as the fibre content is reduced. Although, for the sake of clarity, we only compare the $10 \mu \mathrm{m}$ data in Figure 15 it is clear from Figure 6 that the higher diameter LF samples exhibit a similar behaviour albeit to a somewhat lesser extent. This is a possible indication that the phenomenon is sensitive to the average fibre diameter and may be some explanation as to why this trend has not apparently been observed previously since the body of work in the literature on LFPA appears to focus exclusively on thicker fibres (mainly $17 \mu \mathrm{m}$ diameter products). Examination of Figures 6-8 also reveal that this phenomenon is probably related to the state of the matrix since the transition is only apparent in Figure 6 where the testing took place below the glass transition temperature (Tg) of the matrix of $80^{\circ} \mathrm{C}$. The $\mathrm{Tg}$ of the boiled samples has been estimated at approximately $=-20^{\circ} \mathrm{C}$ and so room temperature testing is well above $\mathrm{Tg}$ [13]. Similarly testing at $150^{\circ} \mathrm{C}$ is well above the DaM Tg of the PA66 matrix. It is not at all clear what causes this fibre diameter dependent brittle behaviour of the LFPA at low fibre contents. Strain 
magnification effects are known to lower the transverse strain to failure of unidirectional laminates. However, the effect observed in Figures 6 and 15 appears to become greater as the fibre content is lowered. This is the opposite effect that might be expected from a strain magnification explanation. This phenomenon where the composite fails at elongations significantly below the elongation to failure of either the fibre or matrix alone is clearly one which requires further detailed investigation.

\section{Conclusions}

The strength and elongation to failure of the injection moulded long glass fibre reinforced polyamide 6,6 composites in this study was shown to be significantly dependent on the residual fibre length, fibre diameter and fibre concentration. Composite strength was found to increase in a non-linear fashion with increasing fibre concentration and to decrease linearly with increasing fibre diameter. The composite strength determined by flexural testing gave values systematically $60 \%$ higher than those values obtained by tensile testing, a phenomenon explained by the Weibull statistical strength theory. Tensile testing results at $150^{\circ} \mathrm{C}$ and after boiling water conditioning showed similar severe loss in strength due to plasticisation of the PA6,6 matrix. The data obtained on the interfacial shear strength in these composites indicated that, with the current sizing systems available for polyamide reinforcement, glass-fibre to polyamide adhesion levels approach the matrix shear strength in the both the DaM and boiling water conditioned state. Consequently improvements to DaM performance of glass reinforced polyamide can more profitably be sought in structure-performance parameters other than adhesion. However, the apparent level of interfacial shear strength was found to decrease significantly with increasing fibre content and to a lesser extent with increasing fibre diameter. Using the results generated on fibre length, concentration 
and diameter and interfacial shear strength, the data on composite strength could be well modelled using the Kelly-Tyson theory with an empirical orientation factor of 0.69 . This factor worked well for both the DaM and boiling water conditioned samples. The DaM tensile elongation behaviour and these LFPA samples exhibited a complex relationship with the variables studied. In particular there was strong evidence of a maximum in the elongation of these materials in the region of $35 \%$ fibre weight content. This maximum appeared to become more prominent with decreasing fibre diameter.

\section{Acknowledgement}

The author gratefully acknowledges the support of Owens Corning with the preparation and testing of the materials used in this study. 


\section{References}

1. Sato N, Kurauchi T, Sato S, and Kamigaito O. Mechanism of fracture of short glass fibre-reinforced polyamide thermoplastic. J. Mater. Sci. 1984:19;1145-1152

2. Sato N, Kurauchi T, Sato S, and Kamigaito O. Reinforcing mechanism by small diameter fiber in short fiber composites. J. Compos. Mater. 1998:22;850-873

3. Horst JJ, and Spoormaker JL. Fatigue fracture mechanisms and fractography of short-glass fibre-reinforced polyamide 6. J. Mater. Sci. 1997:32;3641-3651.

4. Akay M, Barkley D. Fibre orientation and mechanical behaviour in reinforced thermoplastic injection mouldings. J.Mater. Sci. 1991;26:2731-42

5. Laura DM, Keskkula H, Barlow JW, Paul DR. Effect of glass fiber surface chemistry on the properties of glass fiber reinforced, rubber-toughened nylon 6 . Polymer 2002:43;4673-4687

6. Thomason JL. The influence of fibre properties of the performance of glass-fibrereinforced polyamide 6,6. Comp. Sci. Tech. 1999;59:2315-2328.

7. Hassan A, Yahya R, Yahaya AH, Tahir ARM, Hornsby PR. Tensile, Impact and Fiber Length Properties of Injection-molded Short and Long Glass Fiber-reinforced Polyamide 6,6 Composites. J.Reinforced Plastics and Composites 2004;23:969-986.

8. Carlson E, Nelson K. Nylon under the hood: history of innovation. Automotive Engineering 1996;104:84-89.

9. Guyot H. VERTON in Long Fibres. Plast. Mod. Elastom. 1985;37(5):44-45.

10. Toll S, Aronsson C-G. Notched Strength of Long- and Short-Fibre Reinforced Polyamide. Comp. Sci. Tech. 1992;46:43-54.

11. Belbin GR, Staniland PA. Advanced Thermoplastics and their Composites. Philosophical Transactions of the Royal Society of London. Series A, Mathematical and Physical Sciences. 1987;322:451-464. 
12. Bailey RS, Davies M, Moore DR. Processing property characteristics for long glass fibre reinforced polyamide. Composites 1989;20:453-460.

13. Thomason JL. Structure-property relationships in glass reinforced polyamide: 1) The effect of fibre content. Polym.Composites 2006:27:552-562.

14. Thomason JL. Structure-property relationships in glass reinforced polyamide: 2) The effects of average fibre diameter and diameter distribution. Polym.Composites 2007:27:331-343.

15. Thomason JL. Structure-property relationships in glass reinforced polyamide: 3 ) Effects of hydrolysis ageing on the dimensional stability and performance of short glass-fibre reinforced Polyamide 66. Polym.Composites 2007:27:344-354.

16. Thomason J.L. The influence of fibre length, diameter and concentration on the modulus of glass-fibre reinforced polyamide 6,6. submitted to Composites Part A 2008

17. Thomason J.L. The influence of fibre length and concentration on the properties of glass fibre reinforced polypropylene: 5) Injection moulded long and short fibre PP', Composites Part A 2002;33:1641-1652.

18. Fu SY, Lauke B. Effects of fiber length and fiber orientation distributions on the tensile properties of short-fibre-reinforced polymers. Comp. Sci. Tech. 1996;56:1179-1190.

19. Cox HL. The elasticity and strength of paper and fibrous materials. Brit.J.Appl.Phys. 1952;3:72-79.

20. Bader MG, Bowyer WH. An improved method of production of high strength fibre reinforced thermoplastics. Composites 1973;4:150-6

21. Bowyer W.H. and Bader M.G. On the reinforcement of thermoplastics by perfectly aligned discontinuous fibres. J. Mater. Sci., 1972, 7, 1315-1321. 
22. Thomason JL. Interfacial Strength in Thermoplastic Composites - At Last an Industry Friendly Measurement Method ? Composites Part A 2002;33:1283-8.

23. Thomason JL. Micromechanical Parameters from Macromechanical Measurements on Glass Reinforced Polypropylene. Compos.Sci.Technol. 2002;62:1455-68.

24. Thomason JL. Micromechanical Parameters from Macromechanical Measurements on Glass Reinforced Polyamide 6,6. Compos.Sci.Technol. 2001;6:2007-16.

25. Kelly A, Tyson WR. Tensile properties of fibre-reinforced metals. J.Mech.Phys.Solids, 1965;13:329-50.

26. Thomason JL. The influence of fibre length and concentration on the properties of glass fibre reinforced polypropylene: 7. The properties of injection moulded long fibre PP at high fibre content, Composites Part A 2007;38:210-216.

27. Wisnom MR. The relationship between tensile and flexural strength of unidirectional composites. J.Composite Materials 1992;26:1173-1181. 


\section{List of Figure Captions}

Figure 1 DaM tensile strength versus fibre content

Figure 2 Flexural strength versus fibre content

Figure 3 Flexural strength versus tensile strength

Figure 4 Wet tensile strength versus fibre content

Figure 5 Hot tensile strength versus fibre content

Figure 6 DaM tensile elongation versus fibre content

Figure 7 Wet tensile elongation versus fibre content

Figure 8 Hot tensile elongation versus fibre content

Figure 9 DaM IFSS versus fibre content

Figure 10 Wet IFSS versus fibre content

Figure 11 Analysis of orientation factor for Kelly-Tyson equation of tensile strength

Figure 12 DaM tensile strength versus fibre diameter

Figure 13 Wet tensile strength versus fibre diameter

Figure 14 DaM flexural strength versus fibre diameter

Figure 15 Comparison of LF and SF tensile elongation strength versus fibre diameter 


\begin{tabular}{|c|c|c|c|c|c|c|c|}
\hline \multirow[t]{3}{*}{ SF10 } & $\begin{array}{c}\text { Fibre Content } \\
(\% \mathrm{wt})\end{array}$ & & & & & 29.7 & 39.9 \\
\hline & $\begin{array}{l}\text { Fibre Length } \\
(\mathrm{mm})\end{array}$ & & & & & 0.34 & 0.31 \\
\hline & $\begin{array}{l}\text { Orientation } \\
\text { Parameter }\end{array}$ & & & & & 0.78 & 0.77 \\
\hline \multirow[t]{3}{*}{ LF10 } & $\begin{array}{l}\text { Fibre Content } \\
\text { (\%wt) }\end{array}$ & 12.2 & 18.5 & 24.6 & 26.7 & 29.7 & 39.7 \\
\hline & $\begin{array}{l}\text { Fibre Length } \\
(\mathrm{mm})\end{array}$ & 0.89 & 0.85 & 0.60 & 0.74 & 0.52 & 0.45 \\
\hline & $\begin{array}{l}\text { Orientation } \\
\text { Parameter }\end{array}$ & 0.77 & 0.80 & 0.80 & 0.81 & 0.78 & 0.78 \\
\hline \multirow[t]{3}{*}{ LF14 } & $\begin{array}{c}\text { Fibre Content } \\
(\% \mathrm{wt})\end{array}$ & & & 20.6 & 31.0 & 40.5 & 44.1 \\
\hline & $\begin{array}{l}\text { Fibre Length } \\
(\mathrm{mm})\end{array}$ & & & 0.93 & 0.73 & 0.67 & 0.62 \\
\hline & $\begin{array}{l}\text { Orientation } \\
\text { Parameter }\end{array}$ & & & 0.80 & 0.78 & 0.80 & 0.84 \\
\hline \multirow[t]{3}{*}{ LF17 } & $\begin{array}{c}\text { Fibre Content } \\
(\% \mathrm{wt})\end{array}$ & 19.9 & 25.9 & 31.8 & 39.3 & 44.8 & 50.8 \\
\hline & $\begin{array}{l}\text { Fibre Length } \\
(\mathrm{mm})\end{array}$ & 1.19 & 0.98 & 0.85 & 0.82 & 0.56 & 0.51 \\
\hline & $\begin{array}{l}\text { Orientation } \\
\text { Parameter }\end{array}$ & 0.80 & 0.85 & 0.81 & 0.80 & 0.77 & 0.78 \\
\hline
\end{tabular}

Table 1 Characterisation of moulded samples (ref) 


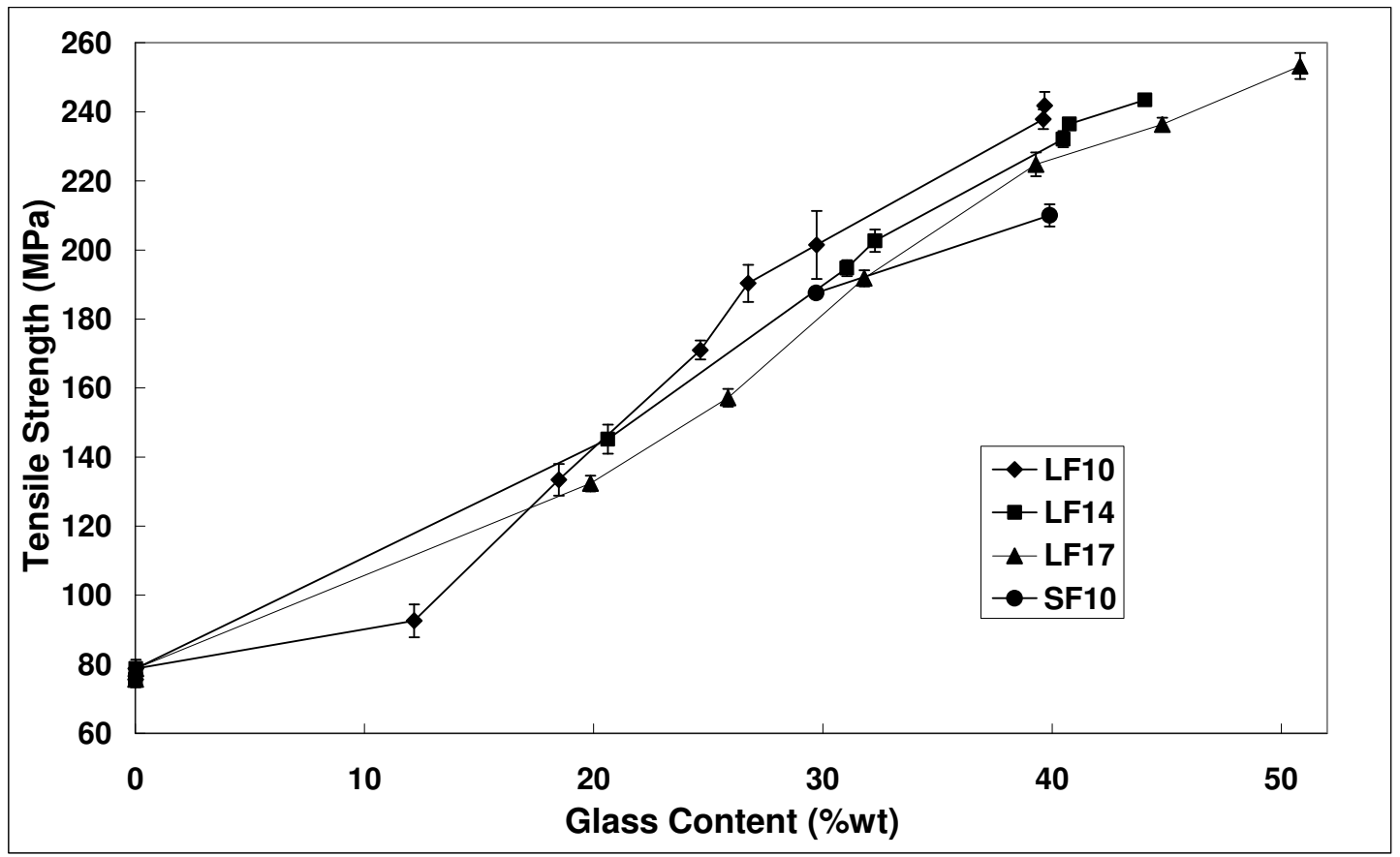

Figure 1 DaM tensile strength versus fibre content

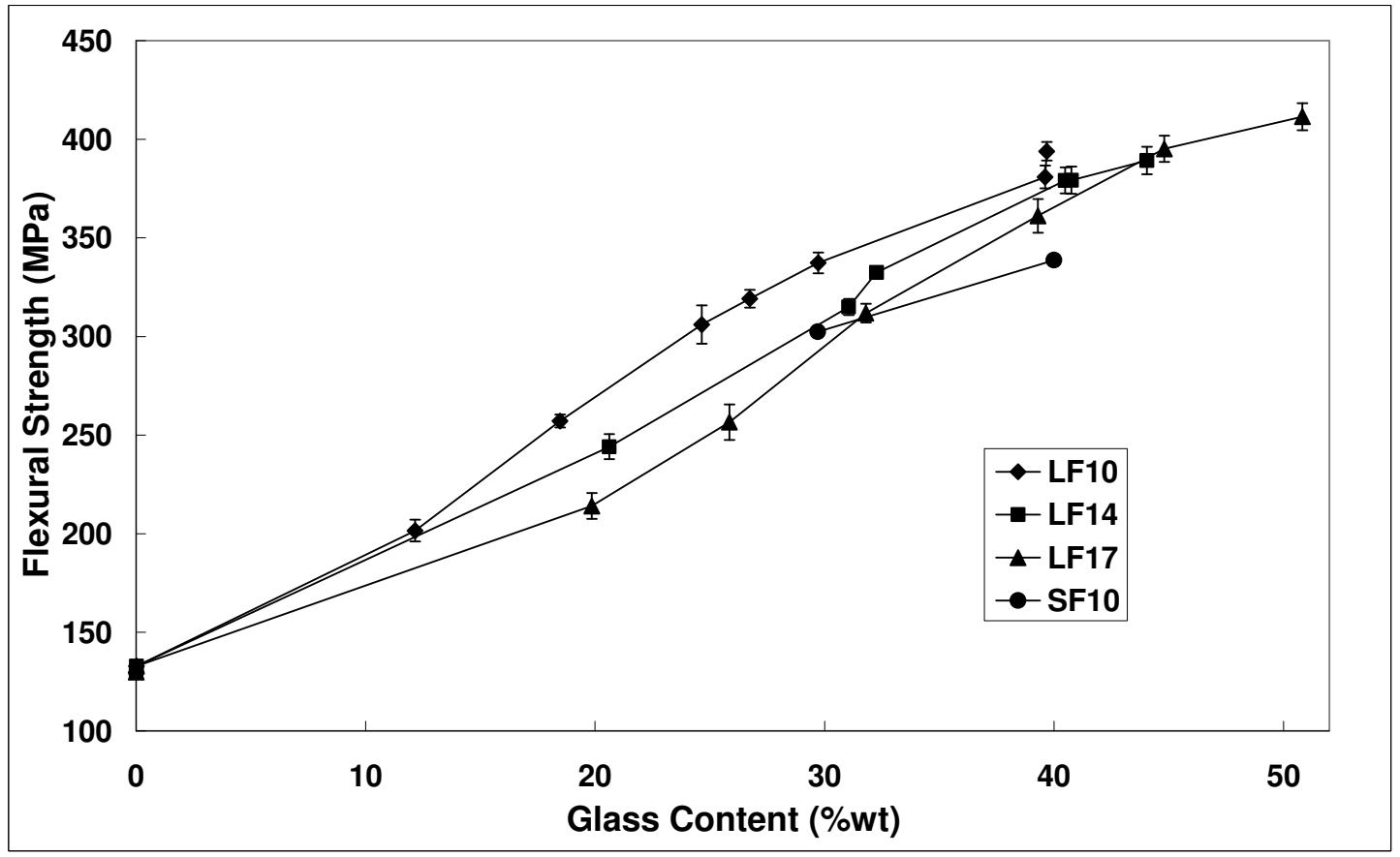

Figure 2 Flexural strength versus fibre content 


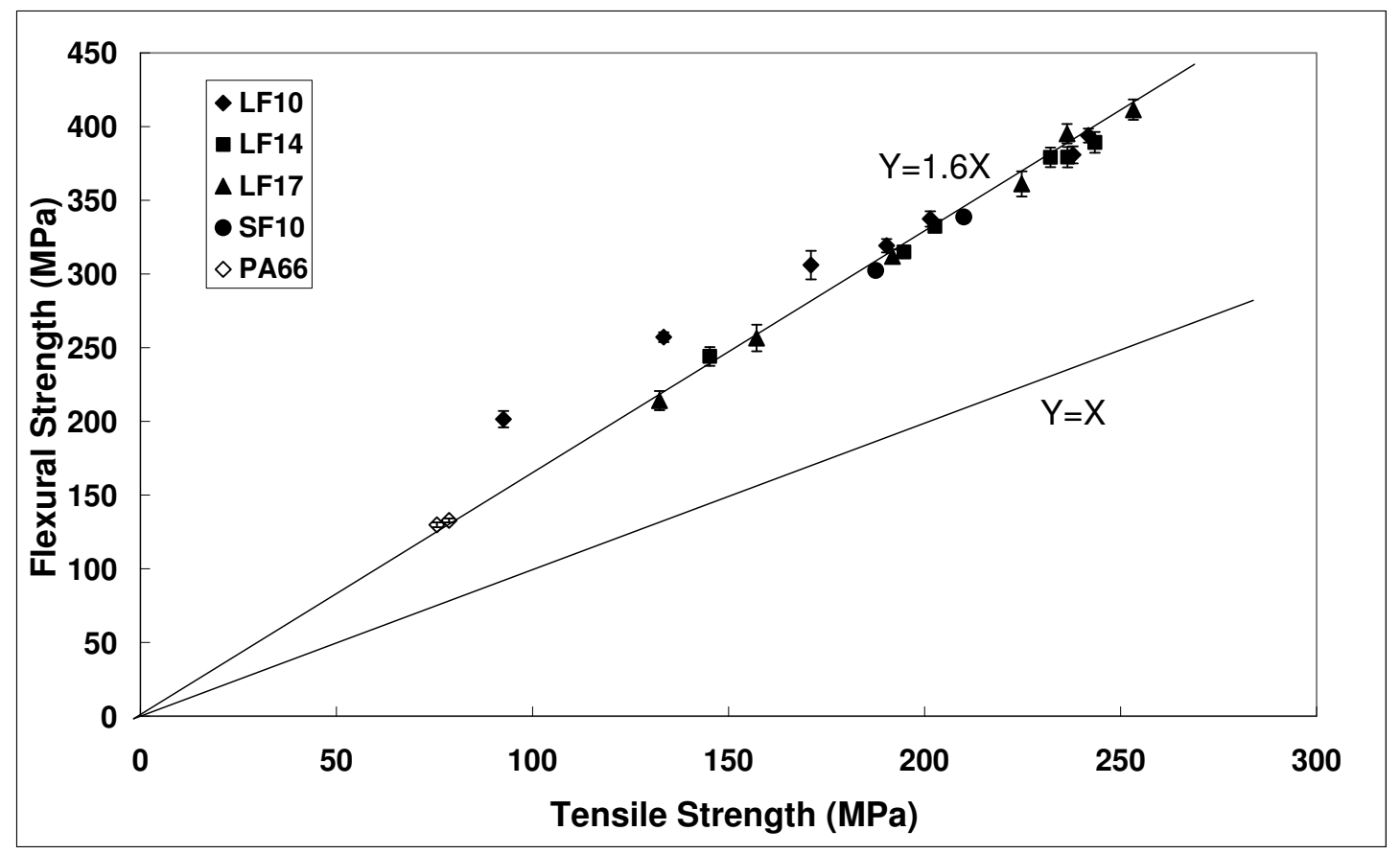

Figure 3 Flexural strength versus tensile strength

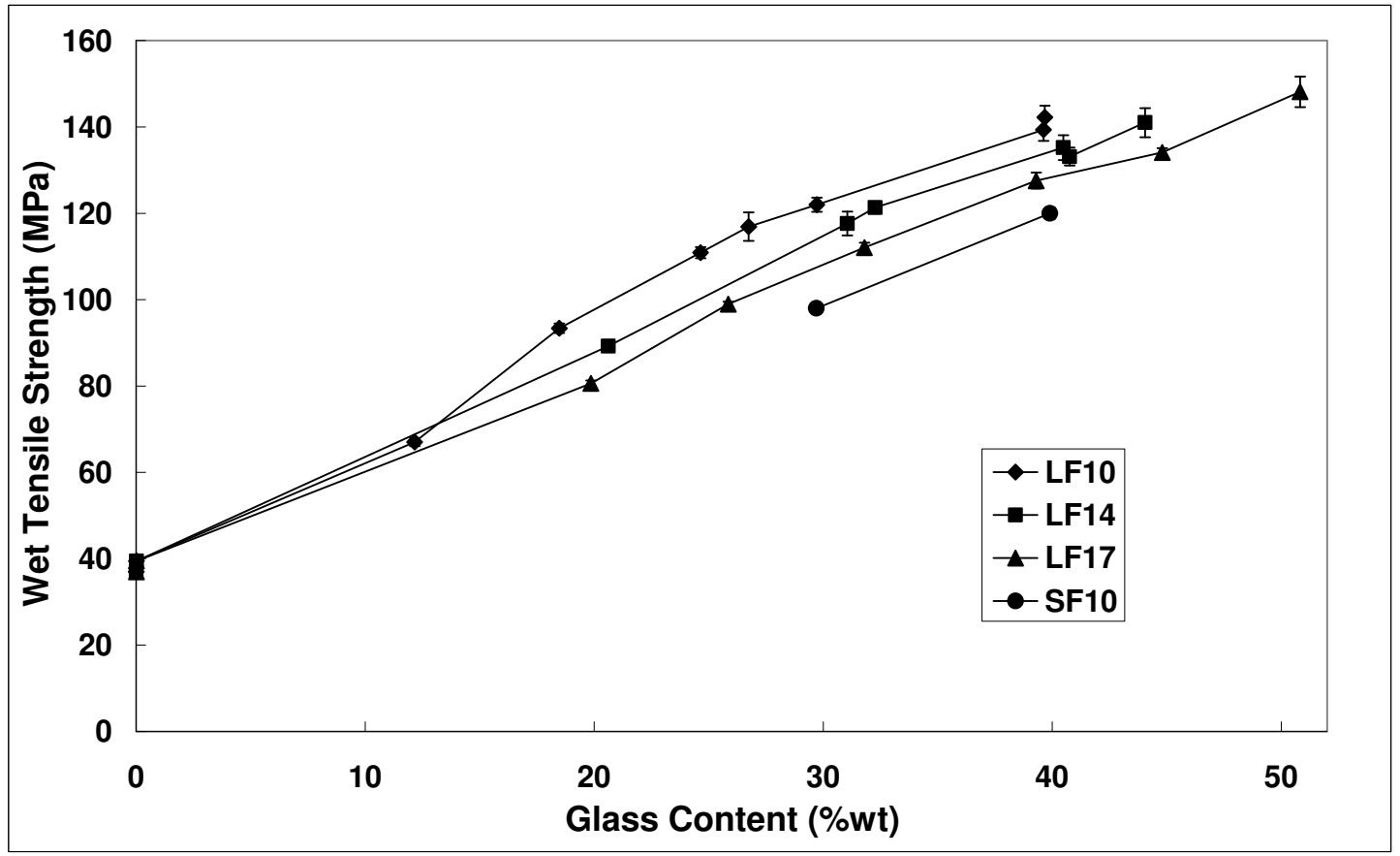

Figure 4 Wet tensile strength versus fibre content 


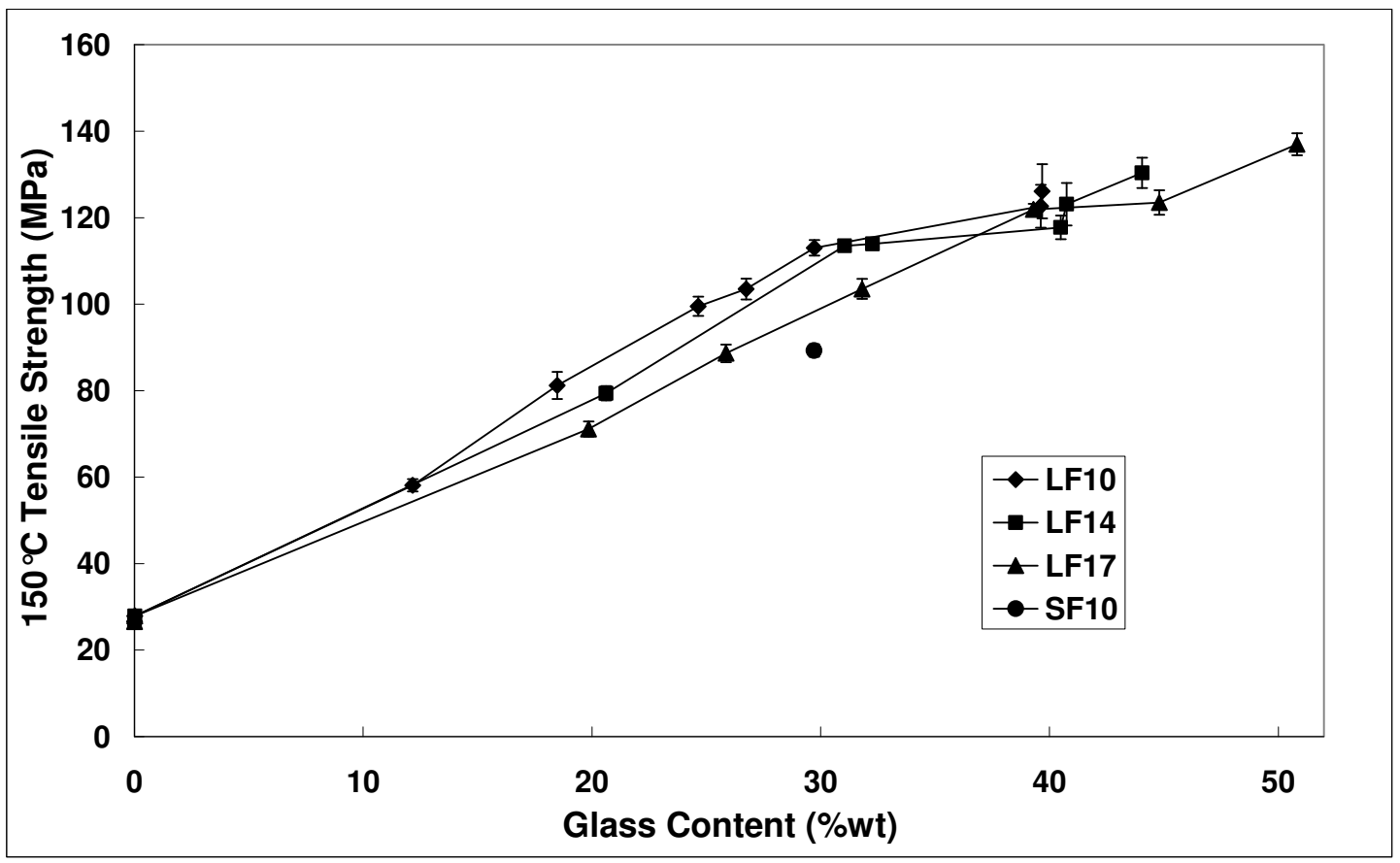

Figure 5 Hot tensile strength versus fibre content

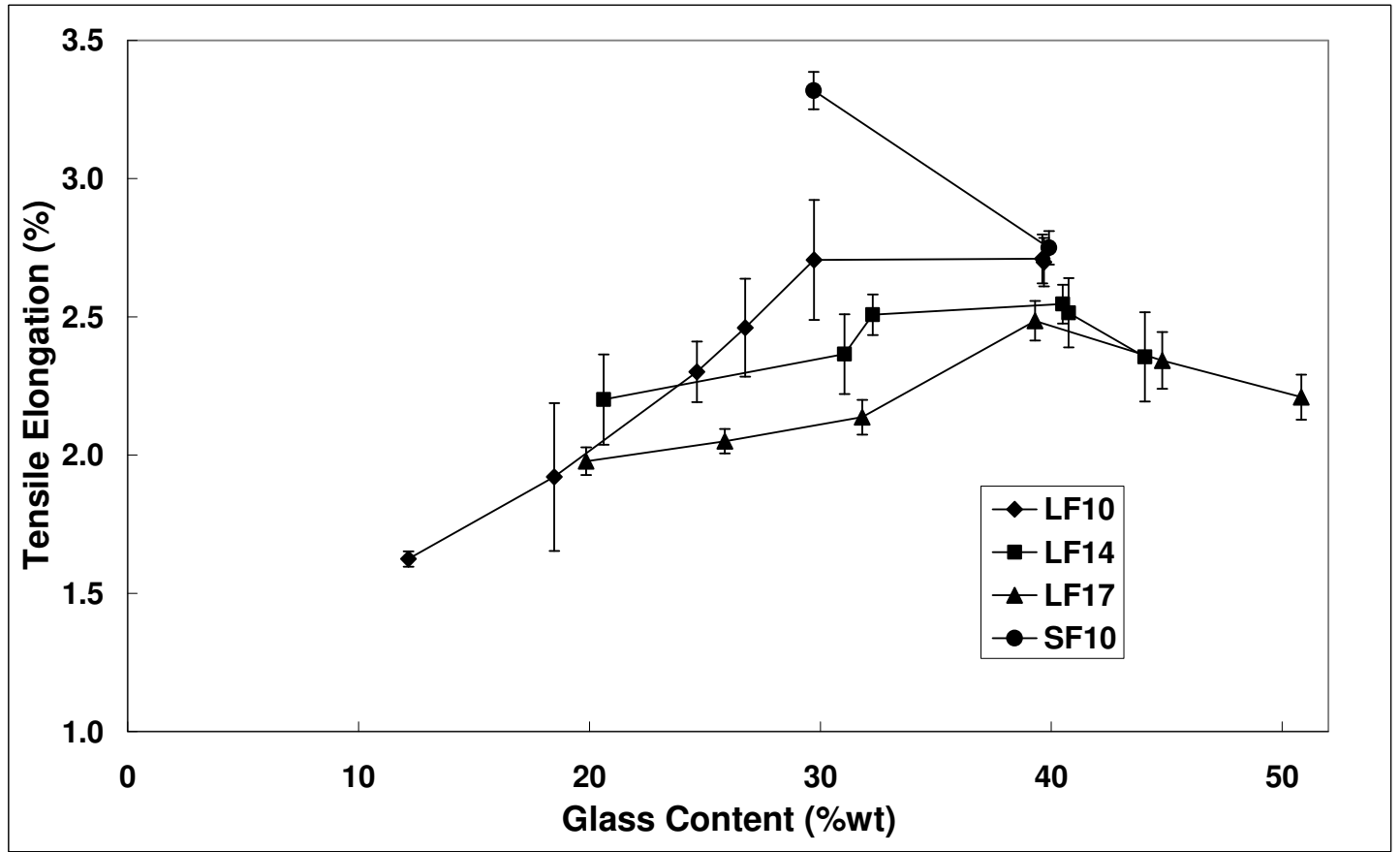

Figure $6 \mathrm{DaM}$ tensile elongation versus fibre content 


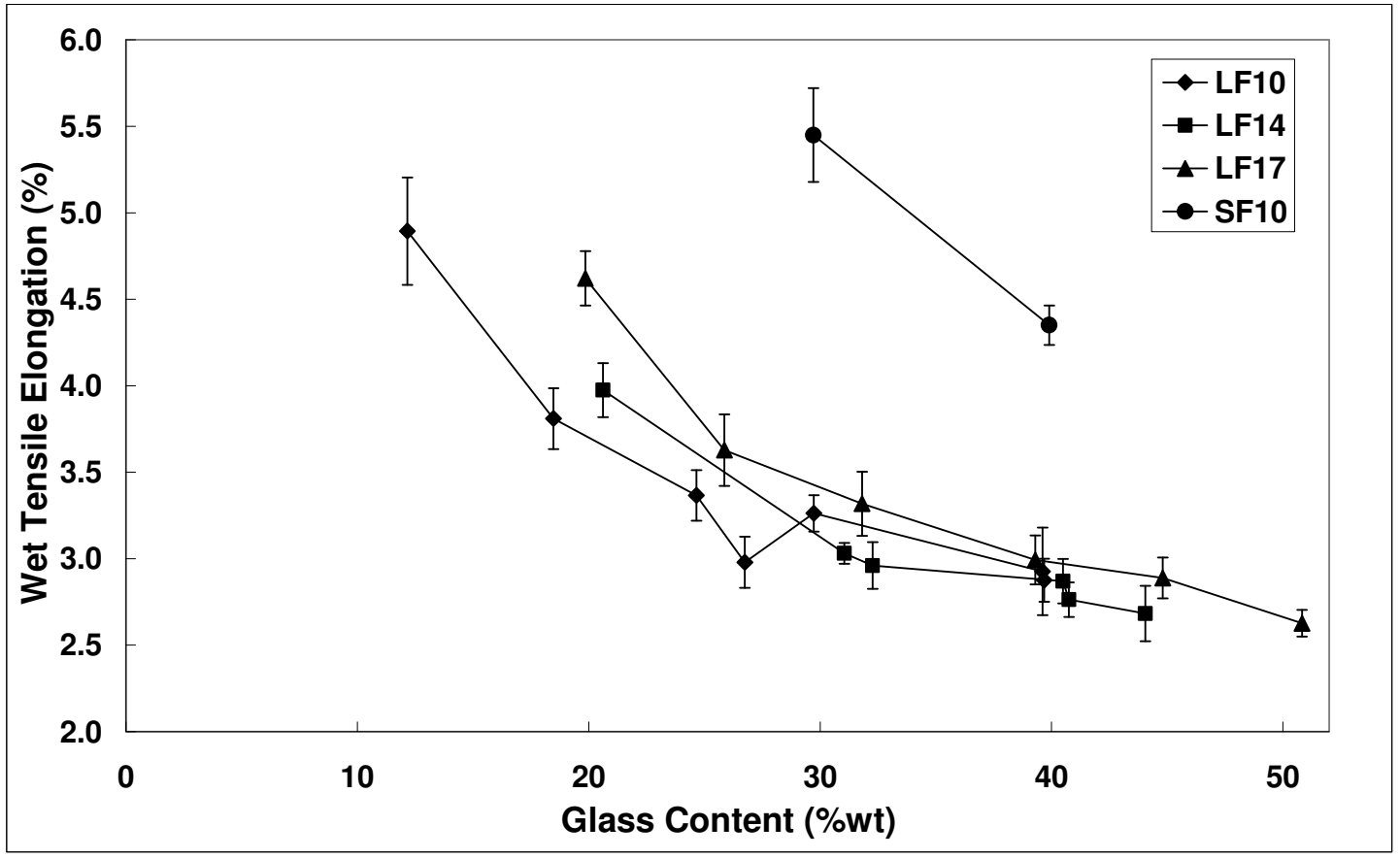

Figure 7 Wet tensile elongation versus fibre content

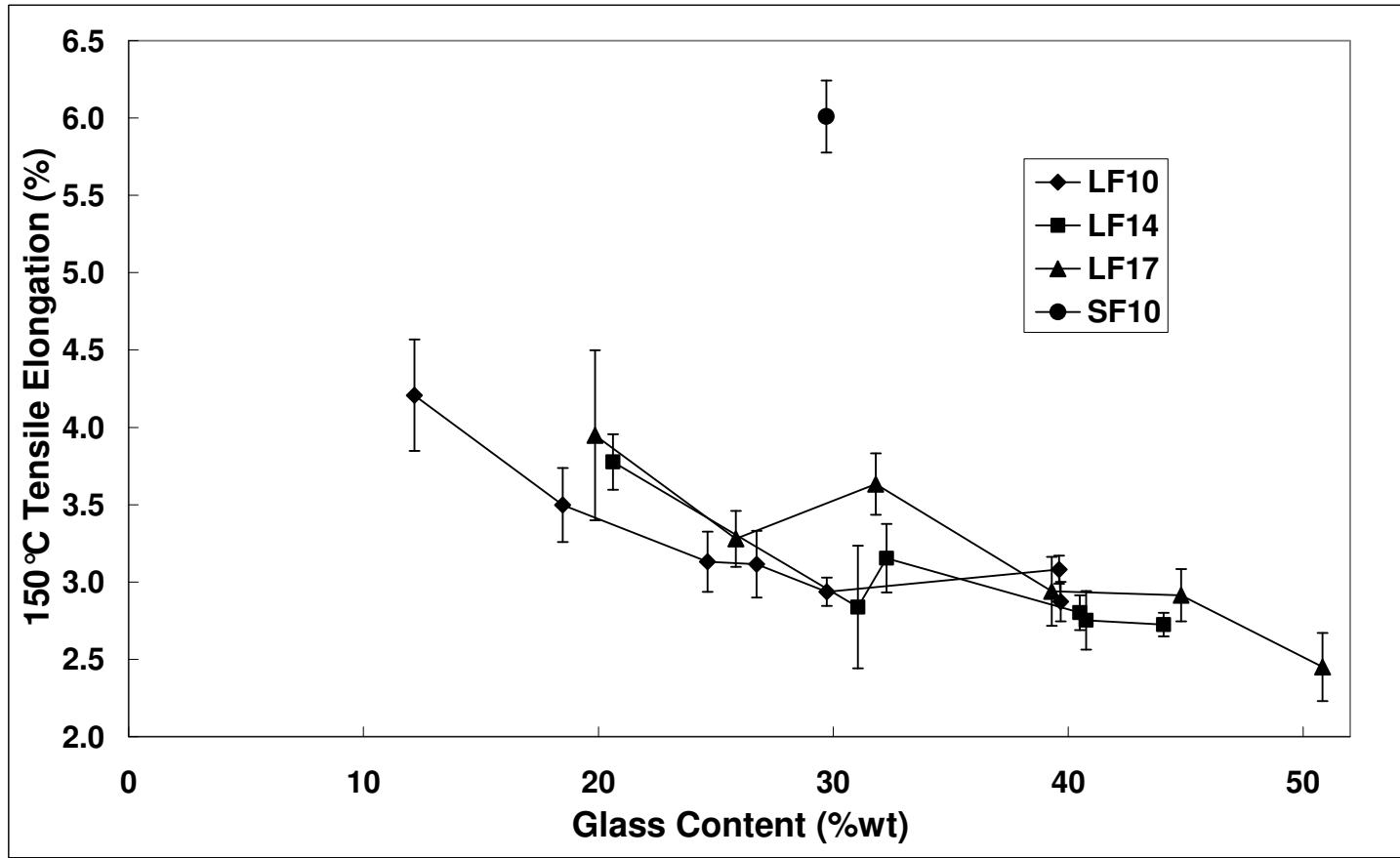

Figure 8 Hot tensile elongation versus fibre content 


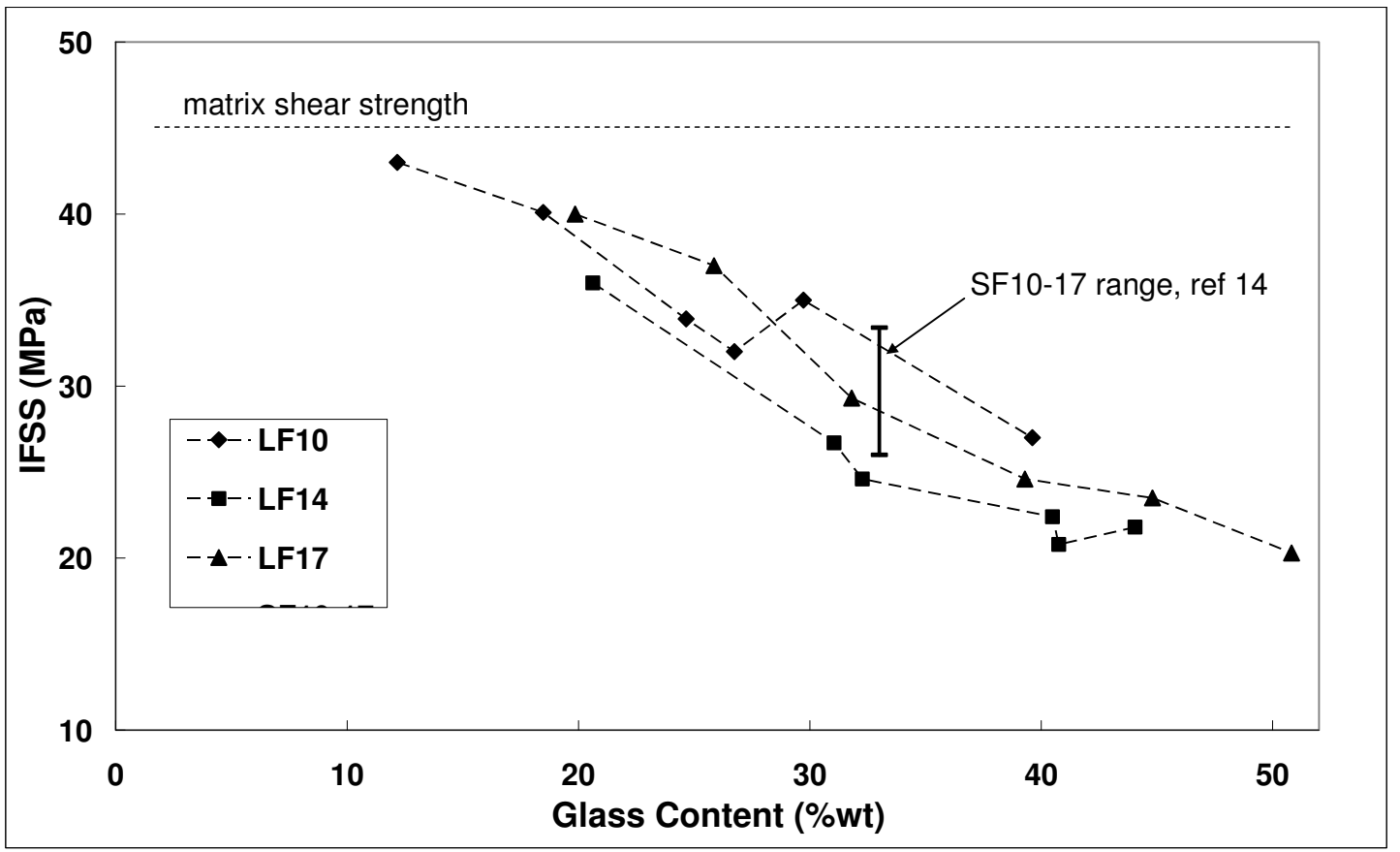

Figure 9 DaM IFSS versus fibre content

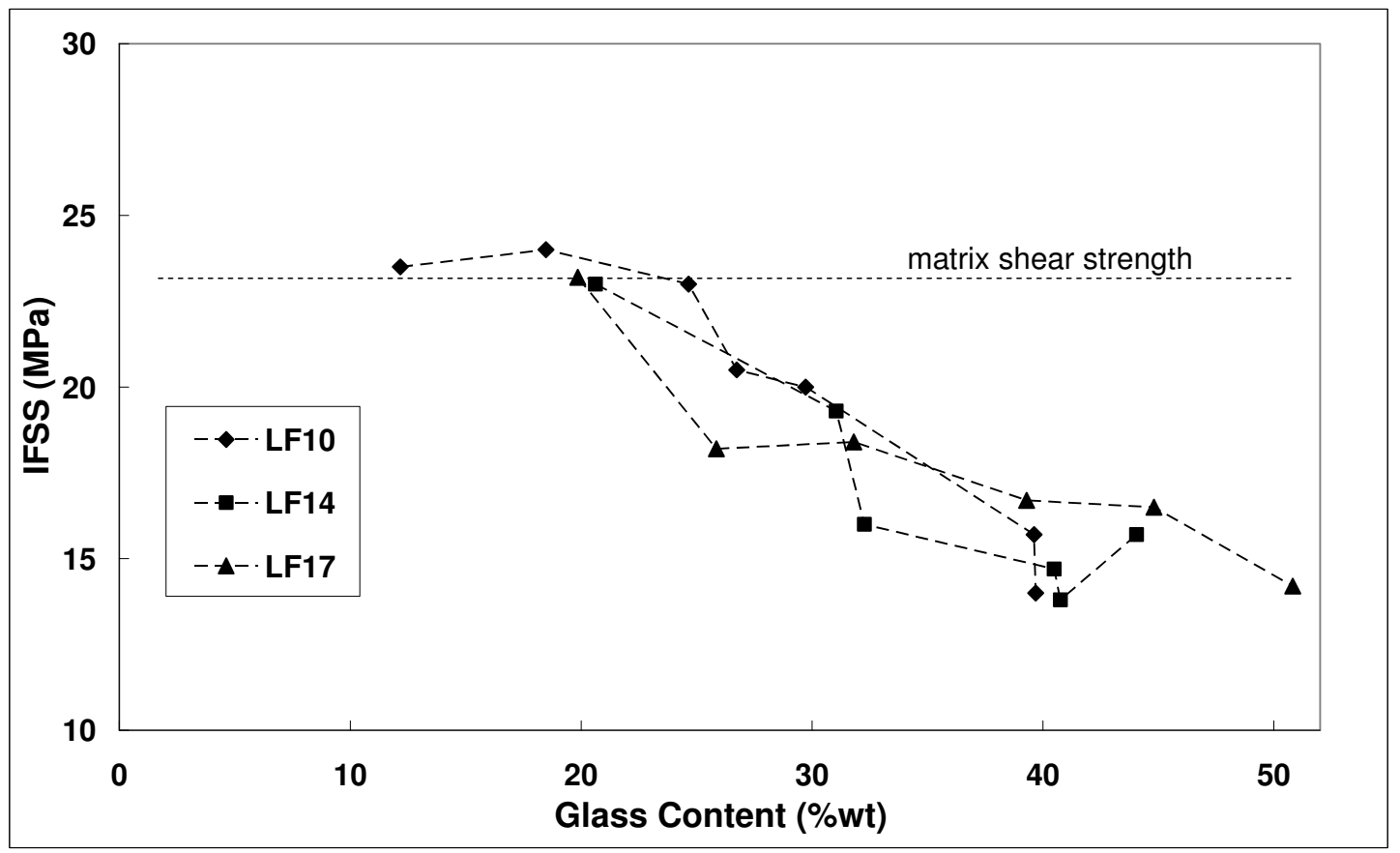

Figure 10 Wet IFSS versus fibre content 


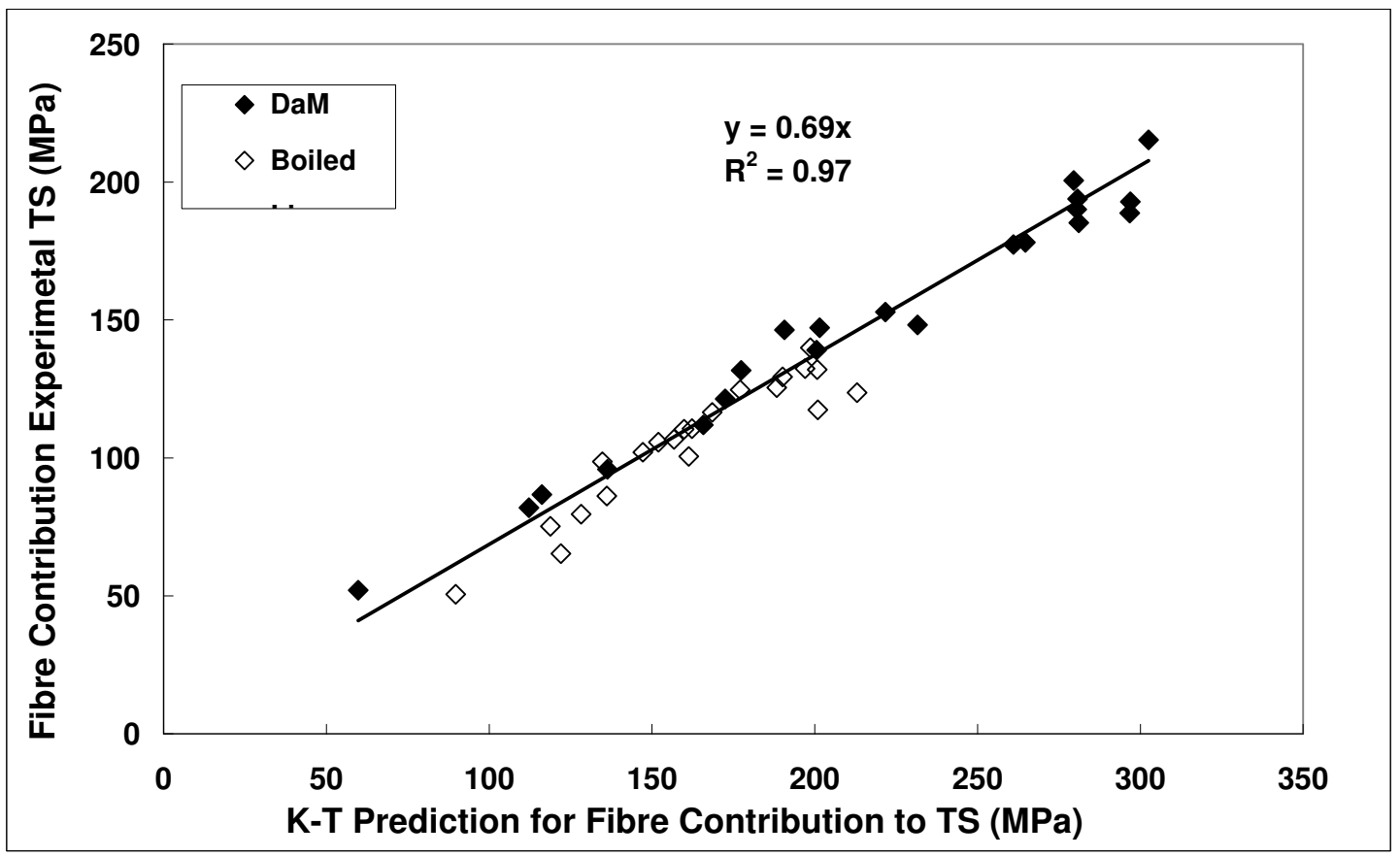

Figure 11 Analysis of orientation factor for Kelly-Tyson equation of tensile strength

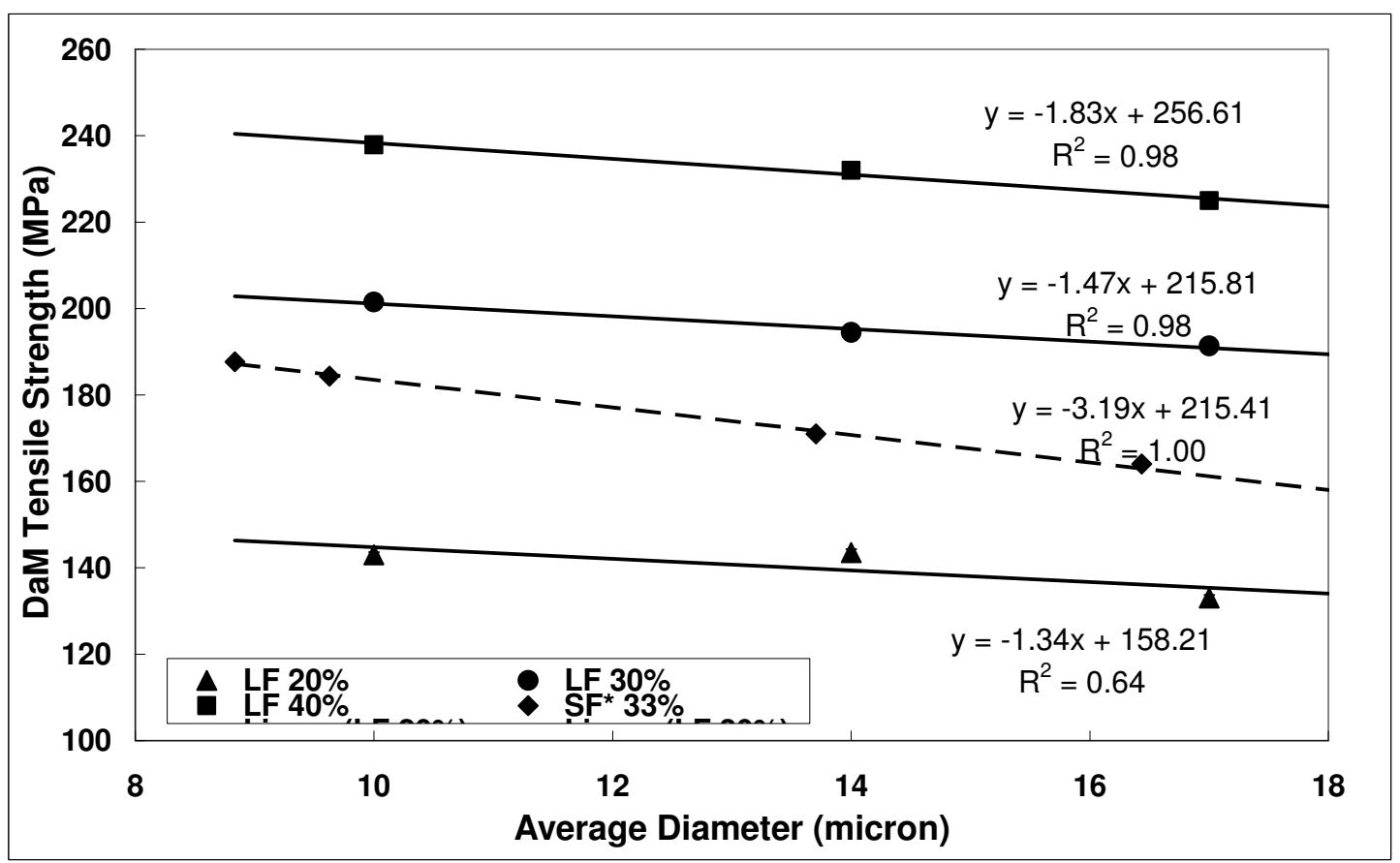

Figure 12 DaM tensile strength versus fibre diameter 


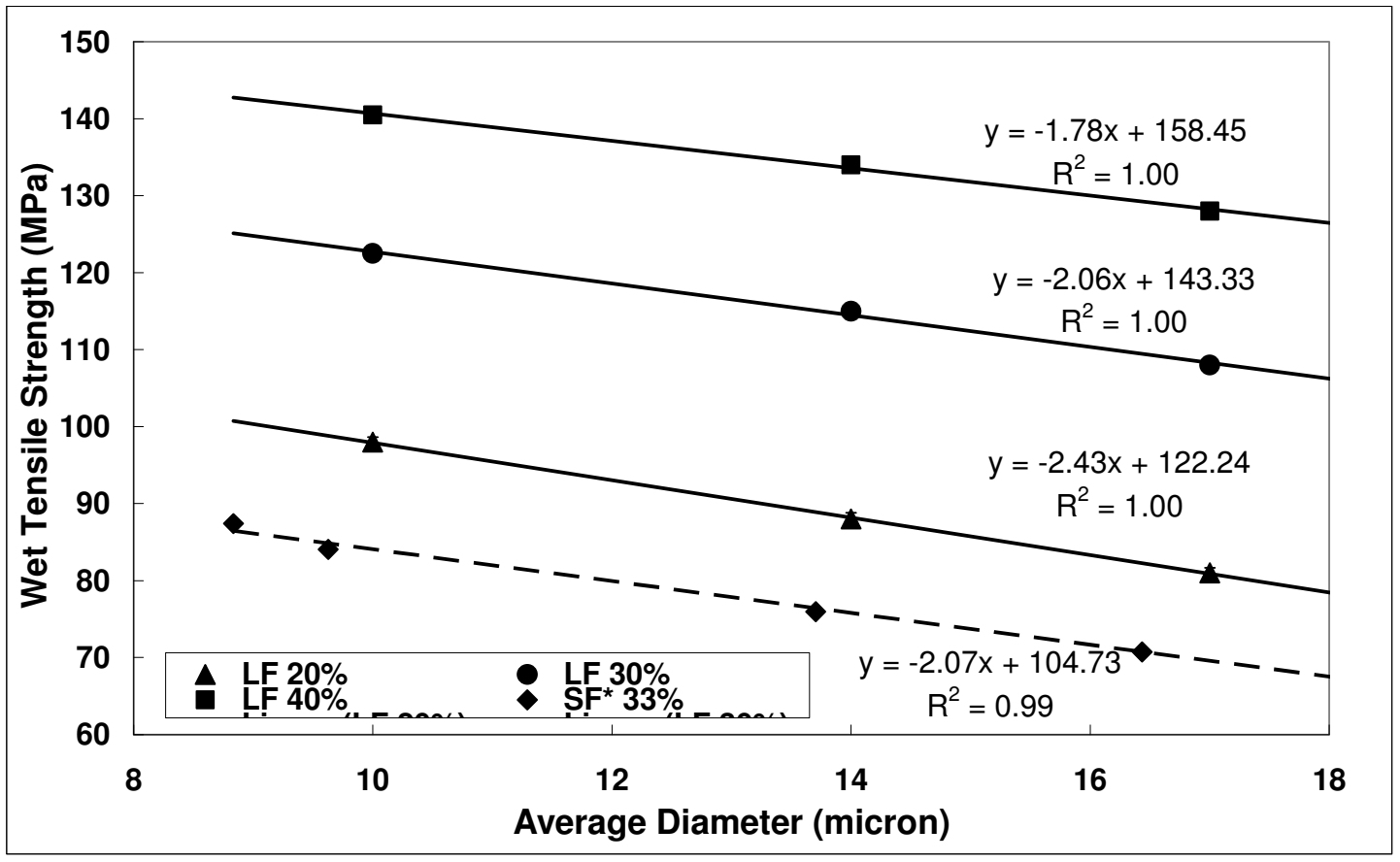

Figure 13 Wet tensile strength versus fibre diameter

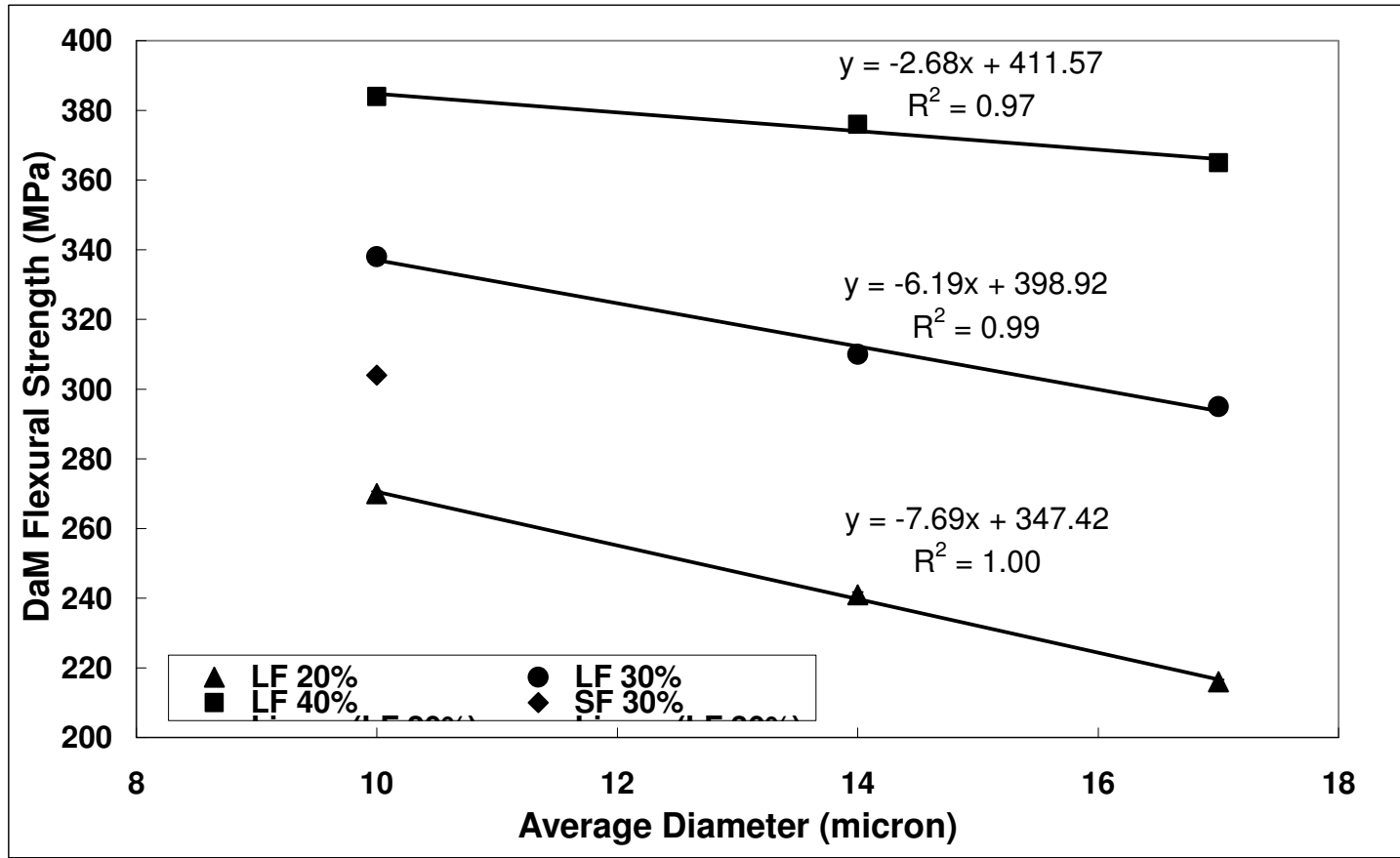

Figure 14 DaM flexural strength versus fibre diameter 


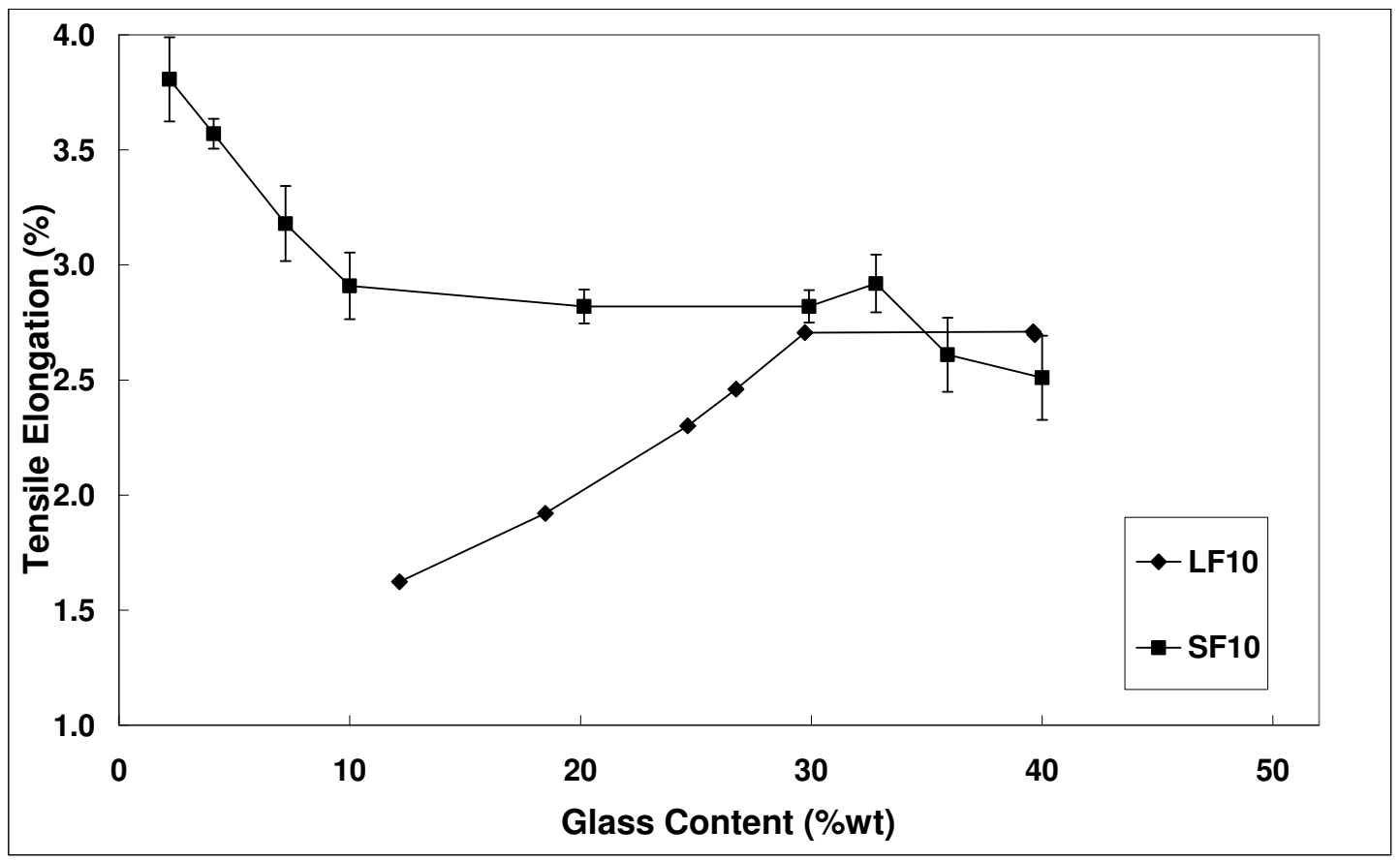

Figure 15 Comparison of LF and SF tensile elongation strength versus fibre diameter 